\title{
面向虚拟试戴系统的人脸位姿估计方法
}

\author{
李明1), 李晋芳1) ${ }^{*}$, 何汉武 ${ }^{2)}$, 蔡嘉鸿 1$)$ \\ 1) (广东工业大学机电工程学院 广州 510006) \\ 2) (广东工贸职业技术学院 广州 510510) \\ (lijinfang@gdut.edu.cn)
}

\begin{abstract}
摘 要: 在虚拟试戴系统中, 虚拟物体需要精准地叠加至实时的人脸图像上, 这决定了虚拟试戴系统的体验感, 其 中的关键技术在于快速准确地估计人脸的实时三维空间位姿. 针对这一技术要求，提出一种实时估计人脸位姿的方 法, 并构建了虚拟试戴原型系统. 首先使用深度相机采集人脸数据, 分析单帧正面图像的彩色和深度信息, 计算人脸 特征点三维数据; 然后结合从视频帧中提取的实时人脸特征点二维数据, 使用直接线性变换法求解出人脸的实时位 姿. 基于消费级深度相机 Astra Mini S 和 Unity3D 引擎开发了原型系统, 并进行了虚拟试戴实验; 使用 Biwi 数据集检 验位姿估计方法的精确度, 并且在不同光照和部分人脸遮挡的条件下测试方法的鲁棒性. 实验结果表明, 文中人脸 位姿估计方法的实时性、精确度和鲁棒性均能满足虚拟试戴系统的体验感.
\end{abstract}

关键词: 虚拟试戴; 人脸位姿估计; 人脸特征点; 深度相机

中图法分类号: TP391.41 DOI: 10.3724/SP.J.1089.2020.18225

\section{A Face Pose Estimation Method for Virtual Try-On System}

\author{
Li Ming $^{1)}$, Li Jinfang ${ }^{1)^{*}}$, He Hanwu ${ }^{2)}$, and Cai Jiahong ${ }^{1)}$ \\ 1) (School of Electronical Engineering, Guangdong University of Technology, Guangzhou 510006) \\ ${ }^{2)}$ (Guangdong Polytechnic of Industry and Commerce, Guangzhou 510510)
}

\begin{abstract}
In the virtual try-on system, virtual objects need to be superimposed on the real-time face image accurately, which determines the experience of the virtual try-on system. The key technology is to estimate the real-time $3 \mathrm{D}$ face pose quickly and accurately. In response to this technical requirement, a method for real-time estimation of the face pose was proposed, and a virtual try-on prototype system was constructed. The method first used a depth camera to collect face data, analyzed the color and depth information of a single frontal image, and calculated the $3 \mathrm{D}$ face feature points; then combined the $2 \mathrm{D}$ real-time face feature points extracted from video frames, and used the direct linear transformation method to solve the real-time face pose. Based on the consumer-level depth camera Astra Mini and Unity3D engine, a prototype system was developed and a virtual try-on experiment was conducted; the accuracy of the pose estimation method was tested using the Biwi data set, and the robustness was tested under different lighting and partial face occlusion conditions. The results show that: the real-time performance, accuracy and robustness of the pose estimation method in this paper can satisfy the experience of the virtual try-on system.
\end{abstract}

收稿日期：2020-02-04; 修回日期：2020-04-25. 基金项目：国家自然科学基金(61300106); 广东省科技计划(2016A040403108). 李明(1994一), 男, 硕士研究生, 主要研究方向为虚拟现实及其可视化技术; 李晋芳(1975一), 女, 博士, 副教授, 硕士生导师, 论文 通讯作者, 主要研究方向为图形图像处理、虚拟现实、增强现实的基础理论和应用技术; 何汉武(1966一), 男, 博士, 教授, 博士生导 师, 主要研究方向为数字化设计与制造、计算机视觉处理、虚拟现实技术及应用; 蔡嘉鸿(1996一), 男, 硕士研究生, 主要研究方向为 虚拟现实及其可视化技术. 
Key words: virtual try-on; face pose estimation; face feature points; depth camera

\section{1 相关工作}

人脸位姿估计指计算机以用户所成图像将脸 部姿态参数化的过程 ${ }^{[1]}$. 其应用范围很广泛, 如虚 拟现实中的虚拟试戴技术、人脸模型重建、康复医 疗辅助系统、自动驾驶和审核认证系统中的活体检 测等. 在虚拟试戴系统中, 为了将虚拟的物体, 如 眼镜、帽子、胡子等准确地叠加至人脸图像上, 达 到虚实融合的显示效果, 关键的问题在于如何快速 准确地获得当前的脸部在三维空间下的位姿状态.

在过去的 20 年里, 计算机视觉领域的研究者 一直在探索脸部位姿估计的方法, 不断推动相关 技术的发展 ${ }^{[2]}$. 目前存在的获取人脸位姿的解决方 案根据使用的数据类型可以分为基于二维彩色图 和基于深度数据 2 种方法. 在二维彩色图研究领 域, Liu 等 ${ }^{[3]}$ 使用大量的二维图片构成的位姿模板, 使用核函数法对位姿模板建立非线性分类模型, 达到了较好的单帧位姿识别效果, 但是需要获取 大量数据生成数据集进行非线性建模, 效率比较 低, 而且不能适应连续帧图像的位姿识别. 针对这 个问题, $\mathrm{Wu}$ 等 ${ }^{[4]}$ 提出卷积神经网络 (convolutional neural network, CNN)级联框架进行人脸检测和姿 态估计, 达到 30 帧/ $\mathrm{s}$ 的实时处理速度, 但其求解出 的在图像二维空间下的位姿缺少一个维度信息. 此外, Bao 等 ${ }^{[5]}$ 使用 CNN 进行解决预测角度范围过 小的问题. Felea 等 ${ }^{[6]}$ 使用深度 CNN 框架训练, 加 上人脸基准点位置等辅助信息，提高了连续脸部 位姿估计的准确率. Lee 等 ${ }^{[7]}$ 使用 $\mathrm{CNN}$ 训练低分辨 率的图像，建立人脸位姿分类模型，可以估计偏航 角和俯仰角，但不能估计翻滚角.

在基于深度数据的方法中, Borghi 等 ${ }^{[8]}$ 使用递 归神经网络对带深度数据的人脸位姿样本进行训 练得出位姿模型. Höffken 等 ${ }^{[9]}$ 使用贝叶斯网络分 割人脸深度图像中的人脸数据, 再通过单级简化 参数(single stage headless, SSH)网络算法处理得到 脸部位姿. Seemann 等 ${ }^{[10]}$ 使用立体相机采集的灰度 和视差图像, 通过视差图像计算深度数据, 将数据 通过神经网络训练位姿估计模型进行预测. 上述 只使用深度数据进行人脸位姿估计的方法, 因为 使用了深度信息, 能在不同光照条件下达到较好 的估计效果，但在应用中采集的深度数据受背景 环境的影响较大, 实时的数据运算对设备性能的 要求较高, 对于多人脸的位姿估计效果比较差.
从目前的情况来看，只结合二维彩色图像或 者深度数据的人脸位姿估计会出现计算数据欠维 度、连续性和实时性不够等问题, 导致这些方法不 适用于实时的虚拟试戴系统. 针对这些问题, 本文 提出了一种基于深度相机的实时估计人脸位姿的 方法: 从二维彩色图像和深度图像快速获取人脸 三维特征点, 然后结合当前人脸图像提取的二维 特征点, 使用直接线性变换法实时进行人脸的位 姿求解. 这种方法能够求得人脸的旋转和位移数 据, 计算速度快, 满足连续及实时性的要求. 此 外，本文基于这种方法，设计并开发虚拟试戴原型 系统, 通过系统中的数据采集功能模块与虚拟试 戴功能模块, 用户能够将虚拟物体快速准确地叠 加显示在人脸图像上, 达到虚实融合的虚拟试戴 效果.

\section{2 本文方法概述}

本文提出的人脸位姿估计方法主要分为 2 个 步骤, 如图 1 所示.

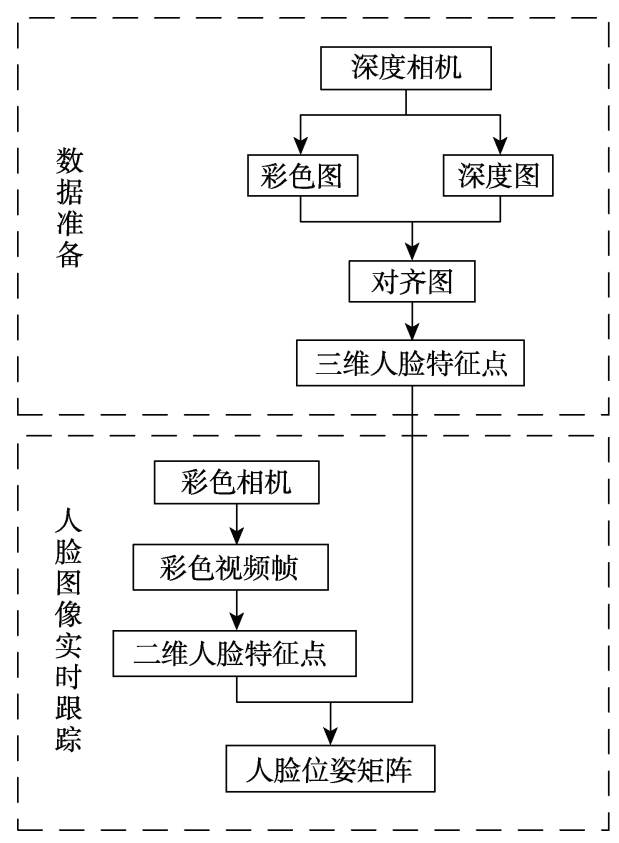

图 1 本文方法框架

(1) 数据准备. 首先使用深度相机采集人脸的 深度数据和彩色图片, 将其进行对齐配准操作, 即 把每个像素坐标的彩色数据与深度数据一一对应, 由深度图可以计算出点云数据; 然后通过人脸特 
征点识别方法获取彩色深度配准图中的特征点数 据, 计算得到在点云数据中的特征点, 完成人脸三 维特征点提取工作.

(2) 人脸图像实时跟踪. 首先使用摄像头实时 获取每一帧人脸图片，对每帧图像使用人脸特征 点提取的方法获取二维特征点像素坐标数据, 得 到的二维特征点数据与数据准备阶段获得的人脸 三维特征点数据进行一一对应; 然后使用直接线 性变换法计算相机的外参矩阵; 最后间接计算出 当前帧人脸的位姿矩阵，完成位姿估计.

\section{3 人脸特征数据的提取}

在本文方法中，数据准备阶段和实时跟踪阶段 需要获取人脸的二维和三维特征点. 本文采用直接 线性变换法求解位姿, 需要至少 6 对二维和三维对 应点. 为了数据获取的稳定和简便，本文选用如图 2 所示人脸的左眼角点、眉心点、右眼角点、鼻尖 点、左嘴角点以及右嘴角点 6 个特征点进行计算.

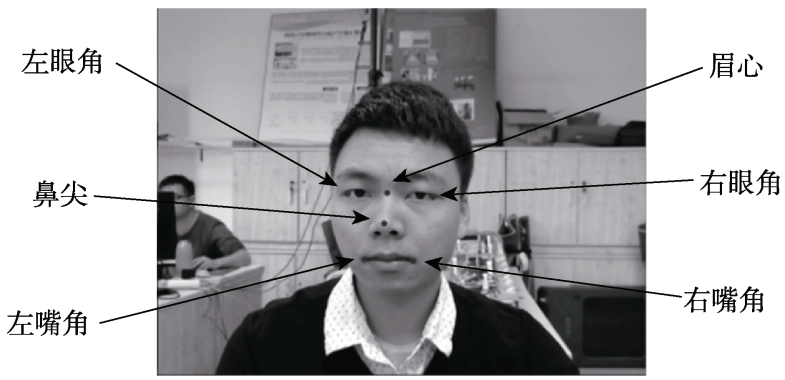

图 2 人脸 6 个特征点选取图

\section{1 二维特征点提取}

人脸二维特征点提取的方法一般分为基于生 成模型和基于判别模型 2 种方法. 基于生成模型的 方法在复杂的人脸姿势变化识别过程中，优化模
型参数时容易陷人局部最优解. 相比之下，约束局 部模型和级联回归等方法采用非参数模型, 可以 更准确地对人脸进行描述. 而与传统图像特征相 比，级联回归方法采用的姿势索引特征对姿势变 化有更好的鲁棒性. 整体而言，基于级联回归的特 征点检测方法对多姿态人脸图像有更好的特征点 检测效果 ${ }^{[11]}$. 因此, 本文采用基于级联回归的人 脸对齐算法梯度提升决策树 (gradient boosting decision tree, GBDT) ${ }^{[12]}$ 对人脸图像进行二维特征点 提取.

GBDT 算法通过建立一个级联残差回归树驱 动人脸形状从当前形状一步一步回归到真实形状, 即由多个弱回归器组合成一个强回归模型, 每一 棵回归树的每一个叶子结点上都存储一个残差回 归量, 当输人落到一个节点上时, 就将残差加到输 人上, 起到回归的目的, 最终将所有残差叠加在一 起，完成人脸对齐. 算法步骤分为训练和测试 2 个 阶段, 如图 3 和图 4 所示. 其中, 人脸形状 $\boldsymbol{S}$ 为人 脸特征点向量, $\boldsymbol{S}=\left(\boldsymbol{x}_{1}^{\mathrm{T}}, \boldsymbol{x}_{2}^{\mathrm{T}}, \cdots, \boldsymbol{x}_{p}^{\mathrm{T}}\right) \in \mathbb{R}^{2 p}, \boldsymbol{x}_{i} \in \mathbb{R}^{2}$, 表示人脸特征点像素坐标. 训练过程中的回归迭 代方程为 $\hat{\boldsymbol{S}}^{(t+1)}=\hat{\boldsymbol{S}}^{(t)}+r_{t}\left(I, \hat{\boldsymbol{S}}^{(t)}\right)$. 其中, $\hat{\boldsymbol{S}}^{(t)}$ 表示 当前估计的人脸特征点向量； $I$ 表示当前图像； $r_{t}$ 为当前训练的弱回归器. 级联回归的关键在于每 一个弱回归器能够基于输人图像的索引特征预测 结果 $\hat{\boldsymbol{S}}^{(t)}$. 为了使弱回归器 $r_{t}$ 达到较好的回归效 果，使用了基于平方和误差的梯度下降算法对回 归器进行训练.

本文使用由 GBDT 算法训练好的模型对人脸 进行二维特征点的识别. 特征点识别效果如图 5 所 示, 脸部的黑点所在位置为识别的特征点位置, 对 于不同的人脸位姿都能准确地识别出特征点.

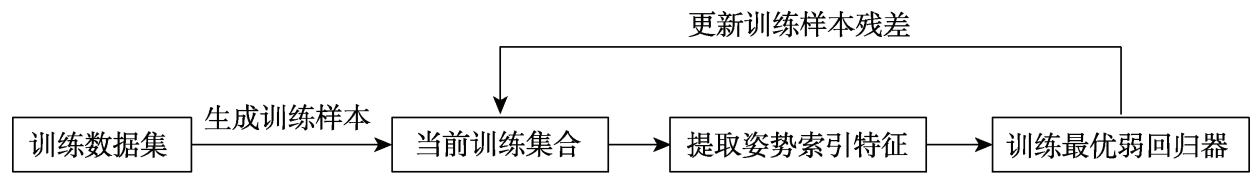

图 3 训练阶段流程图

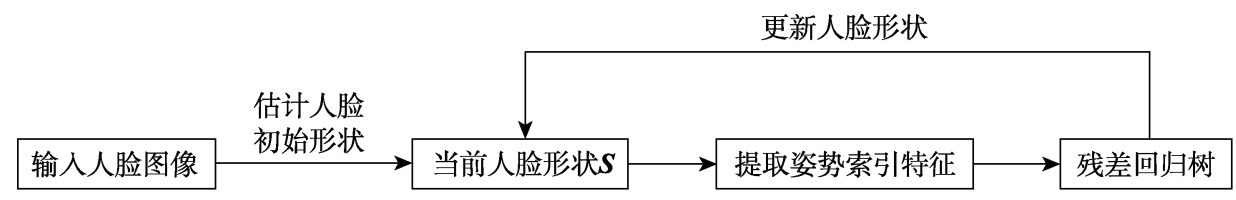

图 4 测试阶段流程图 

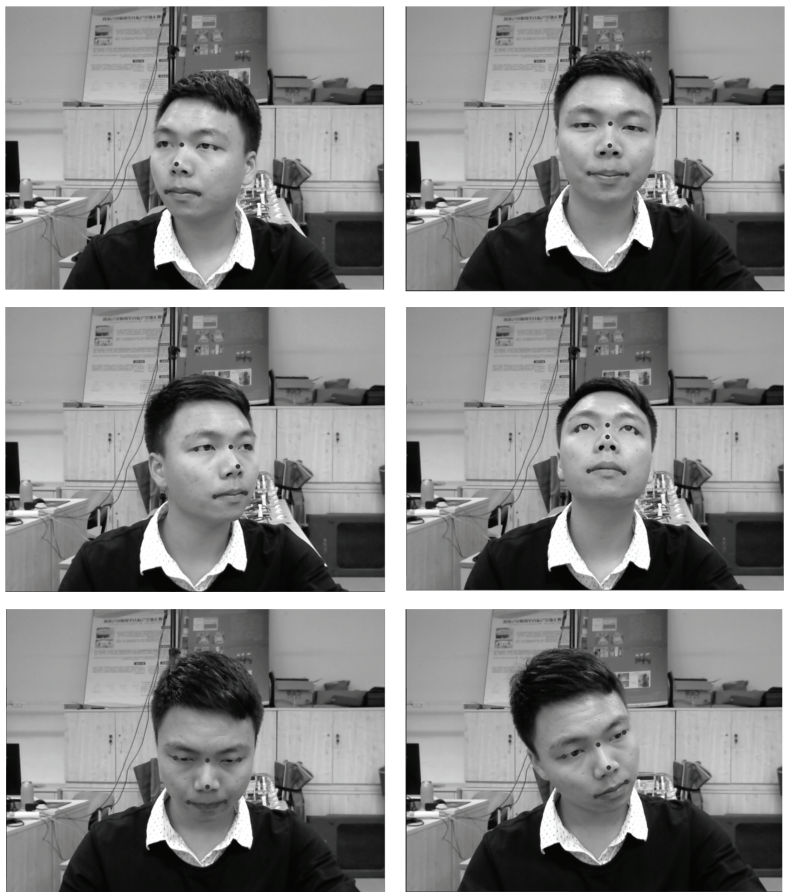

图 5 二维特征点识别效果图

\section{2 三维特征点提取}

在三维特征点的提取过程中, 先将彩色图与 深度图进行配准, 然后提取彩色图中的人脸二维 特征点, 再从配准图中找到与二维特征点对应的 点的深度信息, 结合二维特征点数据和深度信息 计算得到三维特征点集，完成三维人脸特征点的 提取.

世界坐标系下的点映射到二维屏幕点的原理 如图 6 所示. 其中, $u v$ 坐标系表示深度图像所在坐 标系; $O_{\mathrm{w}}$ 为世界坐标系原点; $O_{\mathrm{c}}$ 为相机坐标系 原点.

计算特征点三维数据的过程即将深度图中的 $m$ 点转换为世界坐标系中的 $M$ 点, 转换方程为

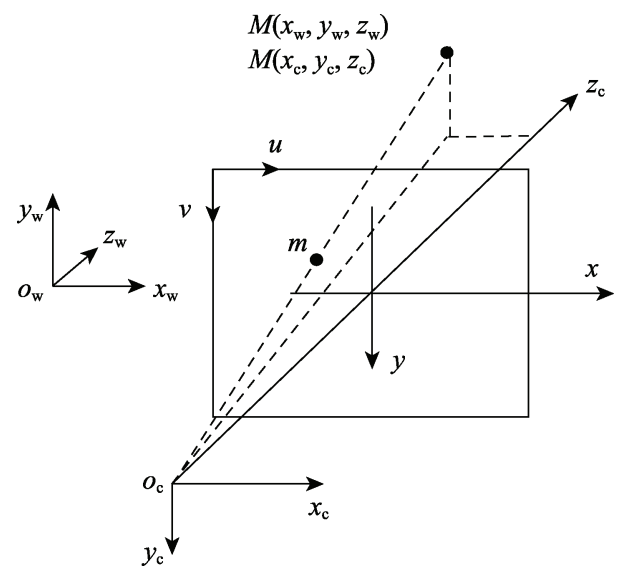

图 6 三维点映射二维点原理图

$$
z_{\mathrm{c}}\left[\begin{array}{c}
u \\
v \\
1
\end{array}\right]=\left[\begin{array}{ccc}
\frac{1}{\mathrm{~d} x} & 0 & u_{0} \\
0 & \frac{1}{\mathrm{~d} y} & v_{0} \\
0 & 0 & 1
\end{array}\right]\left[\begin{array}{ccc}
f & 0 & 0 \\
0 & f & 0 \\
0 & 0 & 1
\end{array}\right]\left[\begin{array}{ll}
\boldsymbol{R} & \boldsymbol{T}
\end{array}\right]\left[\begin{array}{c}
x_{\mathrm{w}} \\
y_{\mathrm{w}} \\
z_{\mathrm{w}} \\
1
\end{array}\right] .
$$

其中, $(u, v)$ 为图像坐标系下的任意坐标点; $\left(u_{0}, v_{0}\right)$ 为图像的中心坐标; $\left(x_{\mathrm{w}}, y_{\mathrm{w}}, z_{\mathrm{w}}\right)$ 表示世界 坐标系下的三维坐标点; $z_{\mathrm{c}}$ 表示相机坐标的 $z$ 轴 值, 即目标到相机的距离; $\boldsymbol{R}$ 和 $\boldsymbol{T}$ 分别为外参矩阵 的旋转矩阵和平移矩阵. 在计算过程中, 将世界坐 标系与相机坐标系重合, 即相机相对于世界坐标 原点没有平移和旋转, 则 $\boldsymbol{R}$ 和 $\boldsymbol{T}$ 分别表示为

$$
\boldsymbol{R}=\left[\begin{array}{lll}
1 & 0 & 0 \\
0 & 1 & 0 \\
0 & 0 & 1
\end{array}\right], \quad \boldsymbol{T}=\left[\begin{array}{l}
0 \\
0 \\
0
\end{array}\right] .
$$

当相机坐标系与世界坐标系的坐标原点重合 时，相机坐标和世界坐标下的同一个物体具有相 同的深度, 即 $z_{\mathrm{c}}=z_{\mathrm{w}}$, 则上述方程进一步转化为

$$
z_{\mathrm{c}}\left[\begin{array}{c}
u \\
v \\
1
\end{array}\right]=\left[\begin{array}{rrr}
\frac{f}{\mathrm{~d} x} & 0 & u_{0} \\
0 & \frac{f}{\mathrm{~d} y} & v_{0} \\
0 & 0 & 1
\end{array}\right]\left[\begin{array}{cccc}
1 & 0 & 0 & 0 \\
0 & 1 & 0 & 0 \\
0 & 0 & 1 & 0
\end{array}\right]\left[\begin{array}{c}
x_{\mathrm{w}} \\
y_{\mathrm{w}} \\
z_{\mathrm{w}} \\
1
\end{array}\right]
$$

采用式(1)可以计算得到从图像点到世界坐标 点的变换公式为

$$
\left\{\begin{array}{l}
x_{\mathrm{w}}=z_{\mathrm{c}} \cdot\left(u-u_{0}\right) \cdot \mathrm{d} x / f \\
y_{\mathrm{w}}=z_{\mathrm{c}} \cdot\left(v-v_{0}\right) \cdot \mathrm{d} y / f \\
z_{\mathrm{w}}=z_{\mathrm{c}}
\end{array}\right.
$$

通过式(2)获取彩色深度配准图中的二维特征 点坐标, 包括左右眼角点、眉心点、鼻尖点以及左 右嘴角点坐标, 将其与深度数据结合计算得到 6 个 特征点相对于相机原点的三维坐标, 求解过程如 图 7 所示.

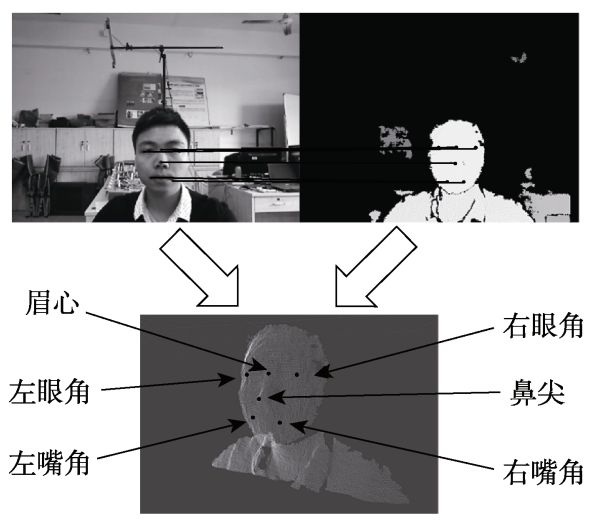

图 7 三维特征点求解过程图 


\section{4. 人脸位姿表达与估计}

\section{1 人脸位姿表达方式}

在三维空间中，描述一个物体的位姿需要考 虑物体在位移和旋转的六自由度方向的参数变化, 常用的位姿表示方法有变换矩阵表示法、欧拉角表 示法以及四元数表示法. 本文使用图 8 所示欧拉角 来表达人脸的位姿，利用俯仰角 $\theta_{y}$ 、偏航角 $\theta_{z}$ 、 翻滚角 $\theta_{x}$ 表达旋转变换. 使用欧拉角表示比较容 易理解刚体旋转的过程，虽然欧拉角容易造成万 向节死锁现象, 即在俯仰角为 $\pm 90^{\circ}$ 时, 第 1 次旋转 与第 3 次旋转使用了同一个轴, 造成系统丢失一个 自由度. 但是本文讨论的人脸旋转变换不会出现 角度为 $\pm 90^{\circ}$ 这种极端的情况, 因此不会出现万向 节死锁现象.

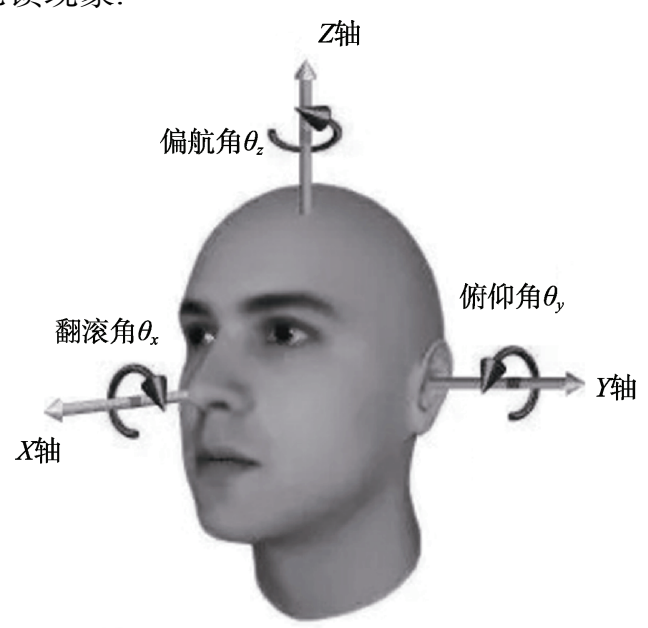

图 8 人脸欧拉角位姿示意图 ${ }^{[13]}$

\section{2 人脸位姿估计}

本文最终求解的人脸位姿是以相机坐标系作 为参照, 便于对求解计算过程的简化以及对结果 进行较为直观的描述，但在求解过程中首先将世 界坐标系原点定于鼻尖点位置, 则可以通过计算 剩余 5 个特征点与鼻尖点的相对位置求得其在此 世界坐标系下的坐标值. 人脸的三维特征点映射 到相机屏幕坐标系的过程与上述求解三维特征点 的计算模型类似，但在现阶段是利用已知 6 组对应 的三维和二维特征点坐标值的条件下求解外参矩 阵 $\boldsymbol{R}$ 和 $\boldsymbol{T}$ ，映射关系为

$$
z_{\mathrm{c}}\left[\begin{array}{c}
u \\
v \\
1
\end{array}\right]=\boldsymbol{M}_{1} \cdot\left[\begin{array}{ll}
\boldsymbol{R} & \boldsymbol{T}
\end{array}\right] \cdot\left[\begin{array}{c}
x_{\mathrm{w}} \\
y_{\mathrm{w}} \\
z_{\mathrm{w}} \\
1
\end{array}\right] .
$$

其中, $M_{1}$ 为 $3 \times 3$ 摄像机的内参矩阵, 包含 $\left(f_{x}, f_{y}, u_{0}, v_{0}\right)$ 参数, 由张正友标定算法 ${ }^{[14]}$ 计算得 到内参矩阵. 位姿矩阵为

$$
\left[\begin{array}{ll}
\boldsymbol{R} & \boldsymbol{T}
\end{array}\right]=\left[\begin{array}{llll}
r_{11} & r_{12} & r_{13} & t_{1} \\
r_{21} & r_{22} & r_{23} & t_{2} \\
r_{31} & r_{32} & r_{33} & t_{3}
\end{array}\right] .
$$

通过 6 组三维与二维特征点数据可以求解方 程组得到 $\left[\begin{array}{ll}\boldsymbol{R} & \boldsymbol{T}\end{array}\right]$ 中的 12 个参数, 进而求得相机在 世界坐标系中这里为人脸鼻尖点作为世界坐标系 原点的旋转与位移量, 即相机坐标系与世界坐标 系的转换方程为

$$
\left[\begin{array}{l}
x_{\mathrm{c}} \\
y_{\mathrm{c}} \\
z_{\mathrm{c}}
\end{array}\right]=\left[\begin{array}{lll}
r_{11} & r_{12} & r_{13} \\
r_{21} & r_{22} & r_{23} \\
r_{31} & r_{32} & r_{33}
\end{array}\right]\left[\begin{array}{l}
x_{\mathrm{w}} \\
y_{\mathrm{w}} \\
z_{\mathrm{w}}
\end{array}\right]+\left[\begin{array}{c}
t_{1} \\
t_{2} \\
t_{3}
\end{array}\right]
$$

由式(3)可以求得在相机坐标系下的世界坐标 系, 即人脸的位姿变化表达为

$$
\begin{gathered}
{\left[\begin{array}{c}
x_{\mathrm{f}} \\
y_{\mathrm{f}} \\
z_{\mathrm{f}}
\end{array}\right]=\left[\begin{array}{l}
x_{\mathrm{w}} \\
y_{\mathrm{w}} \\
z_{\mathrm{w}}
\end{array}\right]=\left[\begin{array}{lll}
r_{11} & r_{12} & r_{13} \\
r_{21} & r_{22} & r_{23} \\
r_{31} & r_{32} & r_{33}
\end{array}\right]^{-1} \cdot\left[\begin{array}{l}
x_{\mathrm{c}}-t_{1} \\
y_{\mathrm{c}}-t_{2} \\
z_{\mathrm{c}}-t_{3}
\end{array}\right]=} \\
{\left[\begin{array}{lll}
r_{11}^{\prime} & r_{12}^{\prime} & r_{13}^{\prime} \\
r_{21}^{\prime} & r_{22}^{\prime} & r_{23}^{\prime} \\
r_{31}^{\prime} & r_{32}^{\prime} & r_{33}^{\prime}
\end{array}\right] \cdot\left[\begin{array}{l}
x_{\mathrm{c}} \\
y_{\mathrm{c}} \\
z_{\mathrm{c}}
\end{array}\right]+\left[\begin{array}{c}
t_{1}^{\prime} \\
t_{2}^{\prime} \\
t_{3}^{\prime}
\end{array}\right]}
\end{gathered}
$$

由式(4)求得人脸相对于摄像机的旋转和位移 表达为

$$
\boldsymbol{R}^{\prime}=\left[\begin{array}{lll}
r_{11}^{\prime} & r_{12}^{\prime} & r_{13}^{\prime} \\
r_{21}^{\prime} & r_{22}^{\prime} & r_{23}^{\prime} \\
r_{31}^{\prime} & r_{32}^{\prime} & r_{33}^{\prime}
\end{array}\right], \quad \boldsymbol{T}^{\prime}=\left[\begin{array}{c}
t_{1}^{\prime} \\
t_{2}^{\prime} \\
t_{3}^{\prime}
\end{array}\right] .
$$

最后将旋转矩阵转化为以欧拉角表示人脸的 旋转状态, 即

$$
\left\{\begin{array}{l}
\theta_{x}=a \tan 2\left(r_{32}^{\prime}, r_{33}^{\prime}\right) \\
\theta_{y}=a \tan 2\left(-r_{31}^{\prime}, \sqrt{\left(r_{32}^{\prime}\right)^{2}+\left(r_{33}^{\prime}\right)^{2}}\right) . \\
\theta_{z}=a \tan 2\left(r_{21}^{\prime}, r_{11}^{\prime}\right)
\end{array}\right.
$$

\section{5 系统搭建与实验结果分析}

基于提出的位姿估计方法，本文设计了一个 虚拟试戴系统，系统整体工作流程如图 9 所示. 在 数据准备和实时跟踪处理 2 个步骤的基础上增加 了数据显示步骤, 构建出整个系统. 


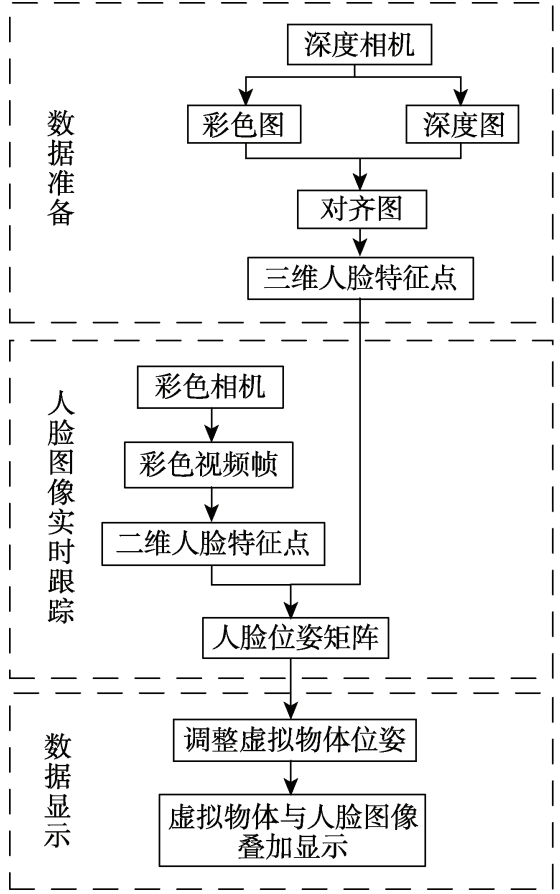

图 9 原型系统工作流程图

\section{1 开发环境与硬件配置}

如图 10 所示, 原型系统分为软件和硬件 2 个 部分. 硬件部分采用的是奥比中光公司生产的 Astra Mini S 深度相机, 可实现彩色和深度数据的 同步采集，相机的深度采集范围为 $0.35 \sim 2.00 \mathrm{~m}$ ， 精度为 $1 \mathrm{~m}$ 内误差 1 3 mm, 其余参数如表 1 所示. 软件系统借助于 Windows 系统的下的 Unity3D 游 戏引擎实现, 使用 C Sharp 语言编程, 能够进行实 时数据获取, 分析计算数据并呈现.

软件系统 Astra Mini S深度相机 用户

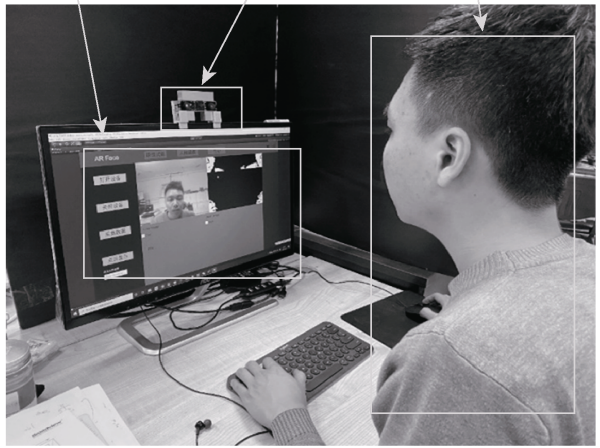

图 10 原型系统图

表 1 深度相机参数表

\begin{tabular}{ccc}
\hline 深度图分辨率/像素 & 彩色图分辨率/像素 & 帧率/ $\left(\right.$ 帧· $\mathrm{s}^{-1}$ ) \\
\hline $1280 \times 1024$ & $1280 \times 960$ & 7 \\
$640 \times 480$ & $640 \times 480$ & 30 \\
$320 \times 240$ & $320 \times 240$ & 30 \\
\hline
\end{tabular}

软件系统主要分为数据采集和虚拟试戴 2 个 功能模块, 如图 11 所示. 在数据采集功能模块下, 用户开启深度相机进行人脸的彩色和深度数据获 取操作, 后续的程序将获取到的彩色图和深度图 对齐, 从彩色图中提取出 6 个人脸特征点 (左右眼 角点、眉心点、鼻尖点以及左右嘴角点)坐标, 在 深度图中获取对应人脸特征点的深度数据, 再结 合第 2 节中的二维图像坐标转换三维坐标点的方 法得出人脸特征点的三维坐标点 (在当前摄像机坐 标系下). 在虚拟试戴功能模块中, 使用彩色摄像 机实时获取用户脸部图像，从每一帧图像中提取 当前帧的 6 个二维特征点, 结合在数据采集功能模 块中获得的 6 个特征点三维坐标, 使用第 3 节中的 位姿矩阵求解方法求解出当前帧人脸相对于摄像 机的位姿. 在显示阶段, 以当前帧人脸图像作为显 示的背景图像，将计算得到的位姿状态(欧拉角表 示)赋予虚拟的佩戴物体, 并使其先于背景图像渲 染出来, 实现虚拟物体叠加至人脸上的显示效果.

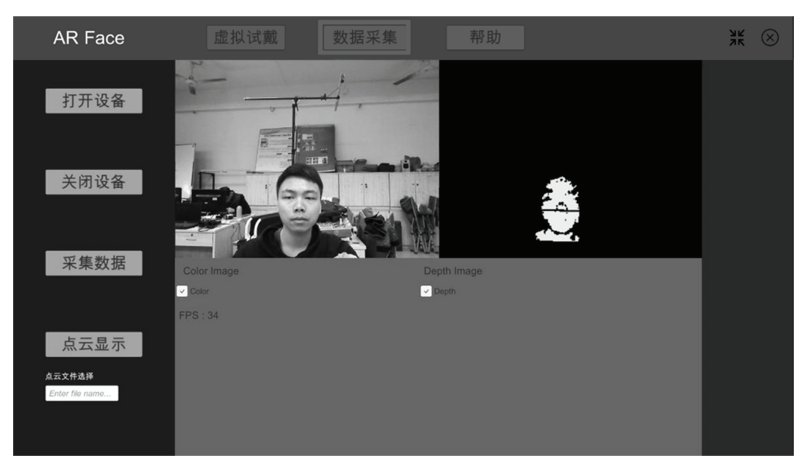

a. 数据采集功能模块

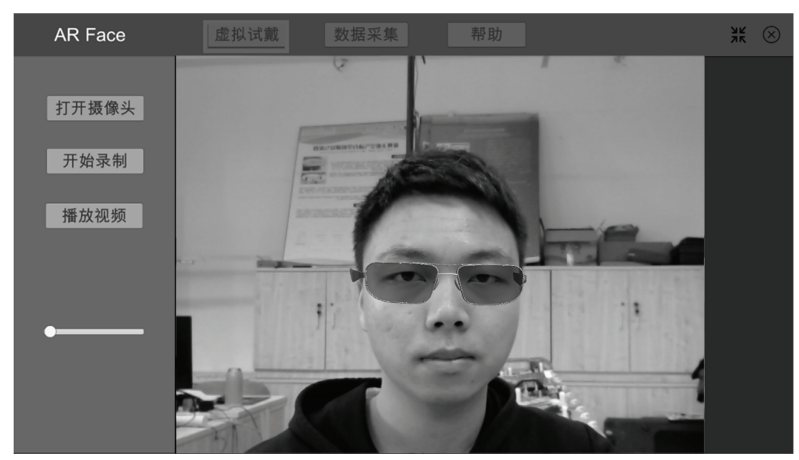

b. 虚拟试戴功能模块

图 11 软件操作界面

\section{2 实验结果分析}

\subsection{1 实时性}

在系统运行测试过程中，使用眼镜作为测试 使用的虚拟试戴物件(也可以是其他物件). 在测试 时, 先使用数据采集功能模块获取到了用户的 6 个 
特征点三维坐标，随后实时获取用户人脸图像计 算出当前人脸的位姿参数并赋子虚拟物体. 图 12 所示为系统运行效果, 使用图 12a 获取的二维特征 点与图 $12 \mathrm{~b}$ 获取的三维特征点计算得到图 $12 \mathrm{c}$ 的当 前人脸位姿, 然后将位姿数据赋予虚拟的物体可
得到图 $12 \mathrm{~d}$ 的虚拟试戴效果. 可以看出，当人脸从 左往右旋转运动时, 虚拟的眼镜能够实时跟随脸 部位姿变化, 佩戴的位置符合实际生活中的眼镜 的佩戴效果. 图 13 和图 14 分别为连续人脸位姿估 计出的旋转和平移变化图. 图 13 和图 14 中的每个
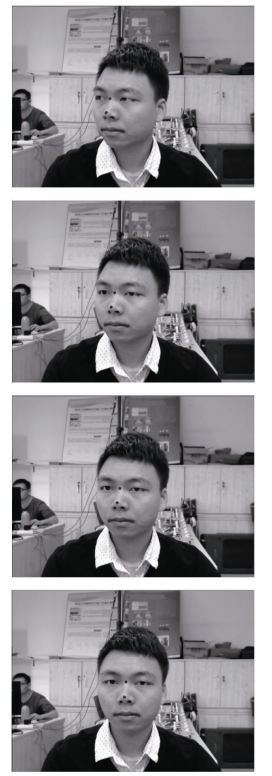

$+$
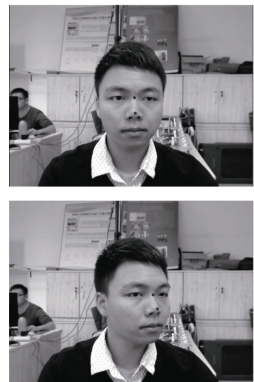

a. 二维特征点数据
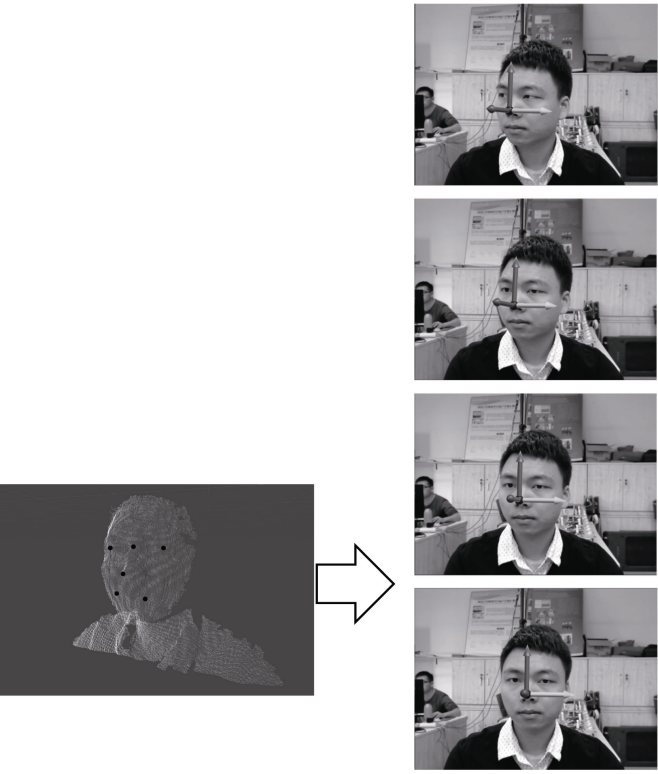

$\square$
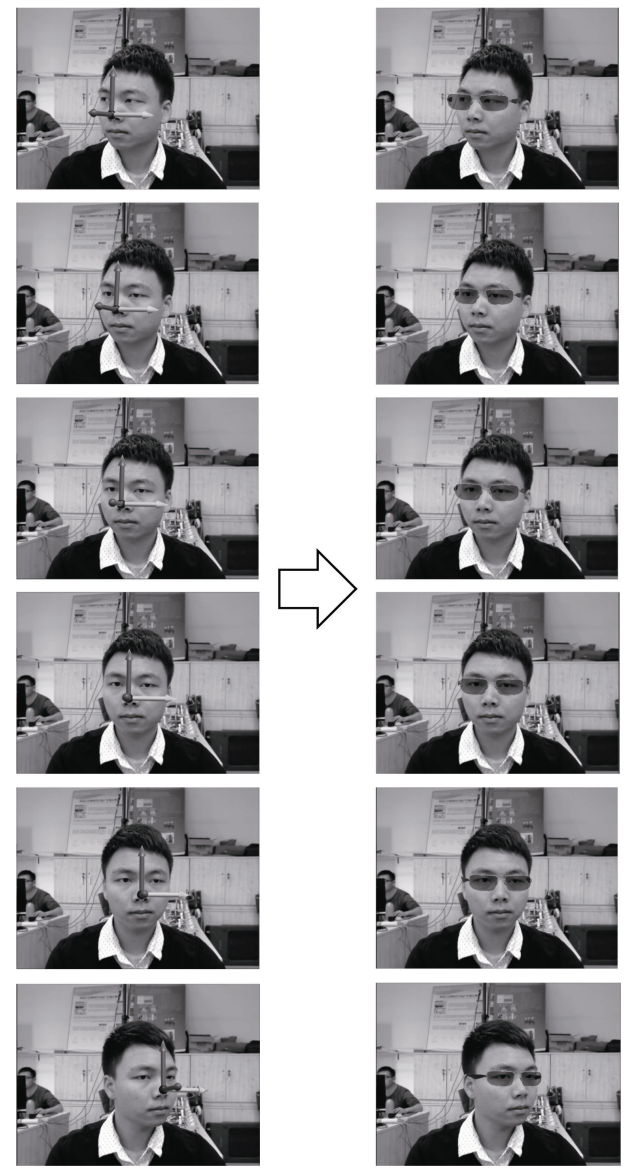

c. 位姿估计结果

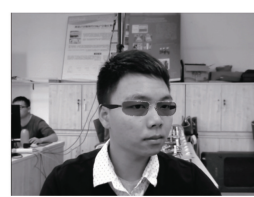

d. 虚拟试戴效果 b. 三维特征点数据

图 12 系统运行效果图

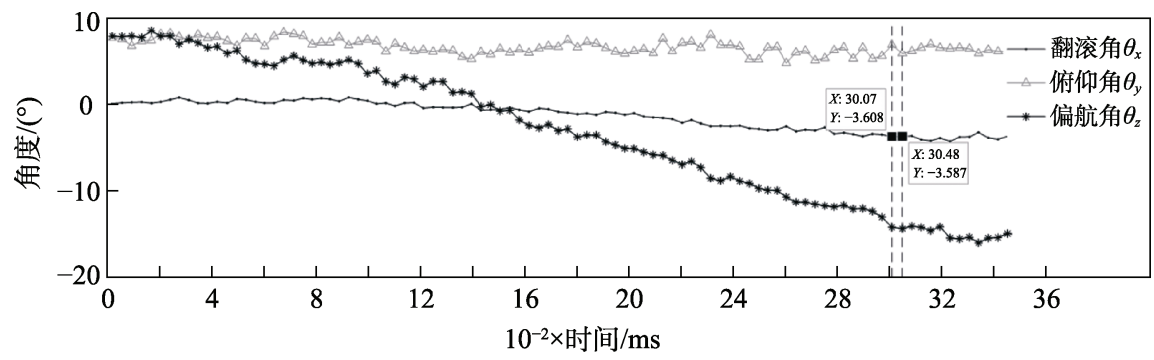

图 13 人脸位姿欧拉角变化图

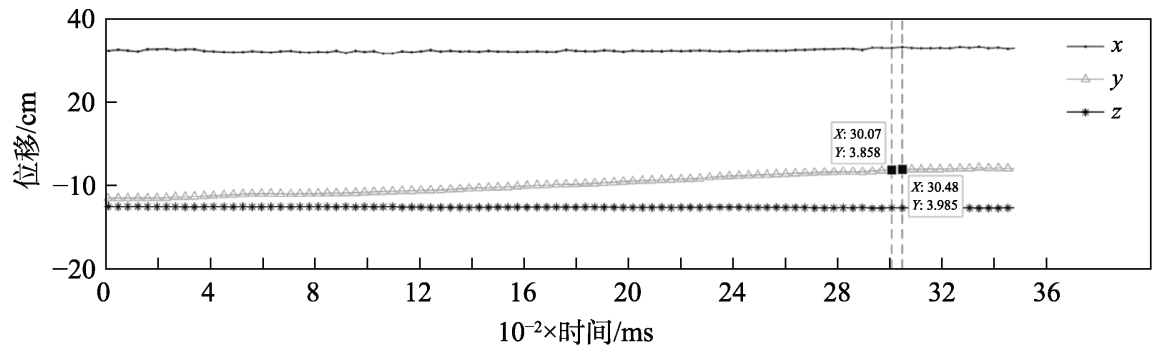

图 14 人脸位姿位置变化图 
数据点为当前时刻计算出的旋转平移参数, 其中 最大的时间间隔为虚线之间的耗时 $41 \mathrm{~ms}$ ，由此可 计算出虚拟佩戴效果渲染帧率可达 24 帧/s，人眼 在实际观影时不会出现卡顿现象，具有实时性.

\subsection{2 精确度}

本文使用 Biwi 数据集对位姿估计方法的精准度 进行校验. Biwi 数据集由 Fanelli 等 ${ }^{[15]}$ 使用 Kinect v2 设备采集, 包含了 20 个人的近 15000 组数据, 每 组数据包括深度数据、彩色图像和图像中人脸的位 姿矩阵信息，适合用于检测本文提出的人脸位姿 估计方法的精确度. 利用数据集中人脸的深度与 彩色信息, 结合本文方法, 可求解出对应的人脸旋 转与平移参数. 通过对比数据集中的人脸位姿信 息, 使用最大误差与平均绝对值误差对人脸位姿 中的翻滚角 $\theta_{x}$ 、俯仰角 $\theta_{y}$ 、偏航角 $\theta_{z}, x$ 轴位移、 $y$ 轴位移和 $z$ 轴位移 6 个参数进行精确度评估, 计 算公式为

$$
\begin{aligned}
& E_{M}=\operatorname{Max}\left\{x^{i}\left|x^{i}=\right| x_{\text {est }}^{i}-x_{\text {real }}^{i} \mid, i \in\{1,2, \cdots, n\}\right\}, \\
& \bar{E}=\frac{1}{n} \cdot \sum_{i=1}^{n}\left|x_{\text {est }}^{i}-x_{\text {real }}^{i}\right| .
\end{aligned}
$$

其中, $E_{M}$ 为对应参数的最大误差; $\bar{E}$ 为平均误差; $x_{\mathrm{est}}^{i}$ 为第 $i$ 组数据中的估计值; $x_{\text {real }}^{i}$ 为第 $i$ 组数据中 的真实值; $n$ 为总数据量. 计算结果如表 2 所示, 旋 转欧拉角中最大的误差为 $1.109^{\circ}$, 最大平均误差为 $0.414^{\circ}$, 最大的位移误差为 $24.628 \mathrm{~mm}$, 最大平均 误差为 $17.364 \mathrm{~mm}$. 结果表明，位姿估计方法的精 确度较高, 满足虚拟试戴系统的要求.

表 2 基于 Biwi 数据集的位姿估计精确度结果

\begin{tabular}{ccccccccc}
\hline \multirow{2}{*}{ 误差 } & \multicolumn{3}{c}{ 旋转参数 $/\left(^{\circ}\right)$} & & \multicolumn{3}{c}{ 位移参数 $/ \mathrm{mm}$} \\
\cline { 2 - 4 } \cline { 6 - 8 } & $\theta_{x}$ & $\theta_{y}$ & $\theta_{z}$ & & $x$ 轴 & $y$ 轴 & $z$ 轴 \\
\hline$E_{M}$ & 0.575 & 0.543 & 1.109 & 20.234 & 19.309 & 24.628 \\
$\bar{E}$ & 0.187 & 0.173 & 0.414 & 11.477 & 14.716 & 17.364 \\
\hline
\end{tabular}

\section{2 .3 鲁棒性}

在验证方法鲁棒性过程中，本文采用在不同 光照条件下以及部分人脸被遮挡的情况来对人脸 位姿估计方法进行鲁棒性分析. 图 15 所示为不同 光照条件下的位姿估计效果，分别使用弱光照、正 常光照和强光照进行实验; 图 16 所示为遮挡部分 人脸后的位姿估计效果. 将坐标系模型位姿与当 前图像中人脸的位姿对比，可以看出本文位姿估 计方法在不同光照或脸部遮挡干扰的条件下具有 较好的鲁棒性.
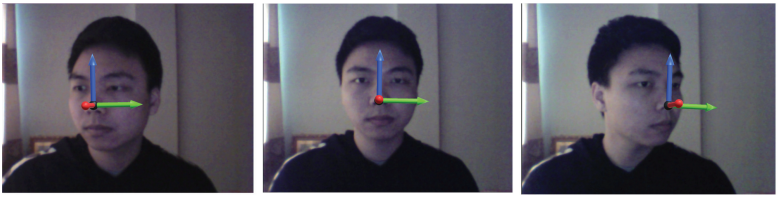

a. 弱光照条件
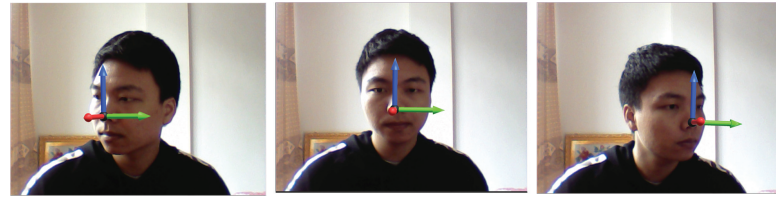

b. 正常光照条件
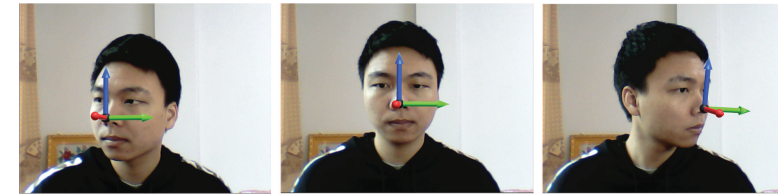

c. 强光照条件

图 15 不同光照条件下的位姿估计结果
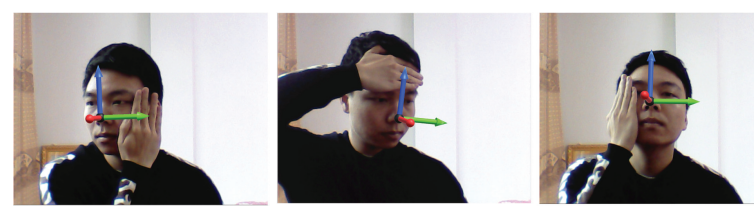

图 16 部分人脸被遮挡的位姿估计结果

\section{6 结 语}

在虚拟试戴系统中，常用的人脸位姿估计方法 使用大量彩色或者深度图像进行训练得出结果, 针 对不同的用户这些方法的训练时间长, 运算复杂, 不适合应用于虚拟试戴系统中的连续帧人脸位姿求 解. 本文针对这些问题, 提出使用深度相机快速获 取人脸特征点, 结合当前人脸图像计算人脸位姿的 方法，并且在该方法的基础上设计了虚拟试戴系 统. 用户通过操作数据采集功能模块与虚拟试戴功 能模块, 能够将虚拟物体快速、准确地显示在人脸 图像上，达到虚实融合的虚拟试戴效果. 最后，通 过实验验证了本文方法具有较好的实时性、精确度 和鲁棒性, 满足虚拟试戴系统的要求. 目前, 这种 人脸位姿估计方法适用于求解人脸偏航角为 $\pm 20^{\circ}$, 翻滚角为 $\pm 45^{\circ}$, 俯仰角为 $\pm 20^{\circ}$ 范围内的位姿, 求解 的范围较小, 因为超过这个角度所需要的特征点会 丢失造成求解失败, 在后续的研究中应增加特征点 提取数量和种类，扩大位姿估计的范围.

\section{参考文献(References):}

[1] Tang Yunqi, Sun Zhenan, Tan Tieniu. A survey on head pose estimation[J]. Pattern Recognition and Artificial Intelligence, 
2014, 27(3): 213-225(in Chinese)

(唐云祁, 孙哲南, 谭铁牛. 头部姿势估计研究综述 $[J]$. 模式 识别与人工智能, 2014, 27(3): 213-225)

[2] Czupryński B, Strupczewski A. High accuracy head pose tracking survey[C] //Proceedings of the International Conference on Active Media Technology. Heidelberg: Springer, 2014: $407-420$

[3] Liu X Z, Li Y W. Face pose estimation based on kernelized maximum separability[J]. Soft Computing, 2018, 22(23): 7825-7831

[4] Wu H, Zhang K, Tian G H. Simultaneous face detection and pose estimation using convolutional neural network cascade[J]. IEEE Access, 2018, 6: 49563-49575

[5] Bao J, Ye M. Head pose estimation based on robust convolutional neural network[J]. Cybernetics and Information Technologies, 2016, 16(6): 133-145

[6] Felea L I, Florea L, Florea C, et al. Head pose estimation using deep architectures $[\mathrm{C}] / /$ Proceedings of the 12th International Conference on Communications. Los Alamitos: IEEE Computer Society Press, 2018: 505-508

[7] Lee S, Saitoh T. Head pose estimation using convolutional neural network[M]. Heidelberg: Springer, 2018

[8] Borghi G, Gasparini R, Vezzani R, et al. Embedded recurrent network for head pose estimation in car[C] //Proceedings of the 28th IEEE Intelligent Vehicles Symposium. Los Alamitos: IEEE Computer Society Press, 2017: 1503-1508

[9] Höffken M, Tarayan E, Kreßel U, et al. Stereo vision-based driver head pose estimation[C] //Proceedings of the IEEE Intelligent Vehicles Symposium. Los Alamitos: IEEE Computer Society Press, 2014: 253-260

[10] Seemann E, Nickel K, Stiefelhagen R. Head pose estimation using stereo vision for human-robot interaction $[\mathrm{C}] / /$ Proceedings of the 6th IEEE International Conference on Automatic Face and Gesture Recognition. Los Alamitos: IEEE Computer Society Press, 2004: 626-631

[11] Huang Yuju. Study of facial feature point detection for multi-pose face based on cascade regression[D]. Guangzhou: South China University of Technology Library, 2016(in Chinese) (黄宇驹. 基于级联回归的多姿态人脸特征点检测算法研究 [D]. 广州: 华南理工大学图书馆, 2016)

[12] Kazemi V, Sullivan J. One millisecond face alignment with an ensemble of regression trees[C]//Proceedings of the IEEE Conference on Computer Vision and Pattern Recognition. Los Alamitos: IEEE Computer Society Press, 2014: 1867-1874

[13] Arcoverde Neto E N, Duarte R M, Barreto R M, et al. Enhanced real-time head pose estimation system for mobile device[J]. Integrated Computer-Aided Engineering, 2014, 21(3): 281-293

[14] Zhang Z Y. Flexible camera calibration by viewing a plane from unknown orientations[C] //Proceedings of the 7th IEEE International Conference on Computer Vision. Los Alamitos: IEEE Computer Society Press, 1999: 666-673

[15] Fanelli G, Dantone M, Gall J, et al. Random forests for real time 3D face analysis[J]. International Journal of Computer Vision, 2013, 101(3): 437-458 